\title{
Applications of laser-induced breakdown spectroscopy for soil analysis, part I: Review of fundamentals and chemical and physical properties
}

\author{
Paulino R. Villas-Boas ${ }^{1}$ (D) | Marco A. Franco ${ }^{2}$ (i) | Ladislau Martin-Neto $^{1}$ | \\ Hero T. Gollany ${ }^{3}$ । Debora M. B. P. Milori ${ }^{1}$
}

${ }^{1}$ Embrapa Instrumentation, São Carlos, Brazil

${ }^{2}$ Institute of Physics, University of São Paulo, São Paulo, Brazil

${ }^{3}$ USDA-ARS Soil and Water Conservation Research, Adams, Oregon

\section{Correspondence}

Paulino R. Villas-Boas, Embrapa Instrumentation, Rua XV de Novembro, 1452, 13560-970, São Carlos, SP, Brazil. Email: paulino.villas-boas@embrapa.br

\section{Funding information}

Conselho Nacional de Desenvolvimento Científico e Tecnológico, Grant/Award Numbers: 312647/2018-2, 403405/2013-0, 434380/2018-0; Fundação de Amparo à Pesquisa do Estado de São Paulo, Grant/ Award Number: 2013/07276-1

\begin{abstract}
Laser-induced breakdown spectroscopy (LIBS) has become a prominent analytical technique in recent years for real-time characterization of soil properties. However, only a few studies of soil chemical and physical properties have been reported using LIBS until recently. The aims of this article are to: (a) provide the basic principles of LIBS for soil analysis and (b) present the use of LIBS for the analysis of soil $\mathrm{pH}$, soil texture and the humification degree of soil organic matter (SOM). The second article will cover soil classification and soil elemental analysis, including plant nutrients, carbon (C) and toxic elements. LIBS is a multi-element analytical technique based on atomic spectroscopy that employs a high-energy laser pulse focused onto a sample surface to create a transient plasma. It is a spectroscopic analytical technique that requires very little or no sample preparation, examines each sample in seconds, and offers a flexible platform for the examination of a broad array of elements in the sample. LIBS also can be used to infer soil chemical and physical properties if a relationship exists between the chemical composition and the soil properties. With proper calibration, LIBS has a great potential for real-time in-field soil analysis and precision farming that could lead to improved soil management and agricultural production, and reduced agricultural environmental impacts.
\end{abstract}

\section{Highlights}

- Laser-induced breakdown spectroscopy (LIBS) is a fast, multi-element analytical technique with great potential for soil characterization.

- Basic principles of LIBS and general description of its use for soil analysis are provided.

- Soil chemical and physical characterization by LIBS are reviewed and compared to other techniques.

- LIBS advantages, limitations and challenges are discussed for soil chemical and physical characterization.

\section{K E Y W O R D S}

humification degree of soil organic matter, precision agriculture, soil analysis, soil $\mathrm{pH}$, soil sensing, soil texture 


\section{1 | INTRODUCTION}

The proper management of soils in agricultural systems can only be achieved by the knowledge of soil chemical, physical and biological properties, including nutrient content, organic matter content, $\mathrm{pH}$, texture, bulk density, waterretention capacity and microbial activity (Tejada \& Gonzalez, 2006). However, obtaining such properties is labour intensive as it requires several techniques, such as: dry combustion for carbon $(\mathrm{C})$, nitrogen $(\mathrm{N})$, hydrogen $(\mathrm{H})$, oxygen (O) and sulphur (S) (Yeomans \& Bremner, 1991); chemical extraction for nutrients (Bray \& Kurtz, 1945; Mehlich, 1984; Olsen, Cole, Watanabe, \& Dean, 1954); inductively coupled plasma (ICP) or atomic absorption spectroscopy (AAS) for multi-element analysis (Houba, Temminghoff, Gaikhorst, \& van Vark, 2000; Huang \& Schulte, 1985; Tüzen, 2003); and the pipette method for soil texture (Liu, Odell, Etter, \& Thornburn, 1966). Most of these techniques require specific sample preparation and take hours to days to analyse a sample; therefore, they are limited for large-scale measurements, especially in the context of precision farming requirements (Borghi, Avanzi, Bortolon, Luchiari Junior, \& Bortolon, 2016). Thus, expedited techniques are a necessity for soil analyses, because they can provide regular monitoring and rapid evaluations of soil properties, even in real time, and could ultimately lead to improved soil management and reduced agroecosystem environmental impacts.

Among recent techniques, laser-induced breakdown spectroscopy (LIBS) is one that has great potential for realtime in-field soil analysis. It is a spectroscopic analytical technique that requires little or no sample preparation, evaluates each sample in seconds and can quantify elemental concentrations of samples when properly calibrated (Cremers \& Radziemski, 2013; Miziolek, Palleschi, \& Schechter, 2006; Noll, 2012). LIBS is also considered a sensitive technique that can detect most elements in the range of $\mu \mathrm{g} \mathrm{g}^{-1}$ (Cremers \& Radziemski, 2013), although it is not as sensitive as ICP, with limits of detection (LOD) in the range of $\mu \mathrm{g} \mathrm{kg}^{-1}$ (Thompson \& Barnes, 1992). In soil samples, LIBS can be used successfully to determine $\mathrm{C}$ content (e.g., see the review by Senesi \& Senesi, 2016), macro- and micronutrients (Díaz, Hahn, \& Molina, 2012; Ferreira et al., 2011; Harris, \& Cremers, D. a, Ebinger, M.H., \& Bluhm, B.K., 2004; Sallé, Cremers, Maurice, Wiens, \& Fichet, 2005) and contaminants (Bousquet, Sirven, \& Canioni, 2007; Senesi et al., 2009). Additionally, LIBS can be used to infer soil chemical and physical properties if a relationship exists between the properties and the chemical composition. For instance, recent studies have demonstrated the ability of LIBS to categorize soil samples (Pontes et al., 2009) and to assess soil pH (Ferreira, Gomes Neto, Milori, Ferreira, \&
Anzano, 2015), the humification degree of soil organic matter (SOM) (Ferreira et al., 2014) and soil texture (VillasBoas et al., 2016), based on the spectral signature of the samples. LIBS can also be easily combined with other techniques, such as Raman spectroscopy, for not only elemental but also molecular analysis (Harmon, Russo, \& Hark, 2013). Because of these advantages, LIBS has become a promising technique that can be coupled with other techniques for direct real-time in-field soil analyses.

LIBS has greatly advanced in recent years due to intensive research on understanding the physical processes involved in the plasma formation, the setup design and data treatment (Noll, 2012; Zorov, Popov, Zaytsev, \& Labutin, 2015). With the advent of double-pulse LIBS systems, the signal acquired has become more stable and reproducible and less affected by matrix effects. The quality of the emission lines has also improved considerably with the new high-resolution spectrometers with intensified charge-coupled device (ICCD) detectors, such as Echelle spectrometers, and time generators operating even at the nanosecond scale, which allows precise control of delay and integration times. In addition, several algorithms are becoming available to correct noise, continuous background, line overlap and matrix effects (Zorov et al., 2015).

A general introduction to the technique can be found in several publications (Cremers \& Radziemski, 2013; W. D. Hahn \& Omenetto, 2010; D. W. Hahn \& Omenetto, 2012; Miziolek et al., 2006; Noll, 2012). Harmon et al. (2013) and Zorov et al. (2015) wrote reviews for geological and environmental applications, including soil and sediment analyses. A review on soil $\mathrm{C}$ analysis using LIBS was written by Senesi and Senesi (2016). Because of its simple setup, LIBS has also been embedded in stand-alone systems, such as that installed in the Curiosity Rover to explore soil on Mars, and made portable for in-field analysis (e.g., handheld devices) (D. Day, Connors, et al., 2015; D. R. Day, Derman, Egan, Soucy, 2015). Recent papers have compared LIBS performance and/or its combined use with other techniques such as near infrared reflectance spectroscopy (NIRS) and visible (Vis)-NIRS in soil applications (Bricklemyer, Brown, Turk, \& Clegg, 2018; Knadel et al., 2017).

The goal of this review article (Part I) is to provide the basic principles and general description of the use of LIBS for soil chemical and physical analysis, including soil $\mathrm{pH}$, soil texture and humification degree of SOM. Here, reference or new techniques will be compared to LIBS along with their advantages and disadvantages. In Part II of this review (Villas-Boas et al., 2019, this issue), soil elemental analysis, including $\mathrm{C}$, nutrients, toxic elements and soil classification, will be presented. 


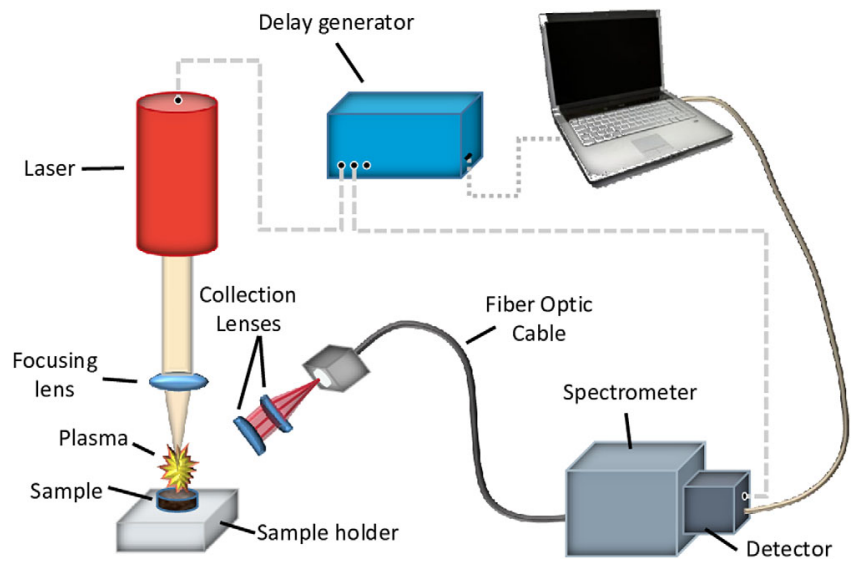

FIG URE 1 A typical laser-induced breakdown spectroscopy (LIBS) setup

\section{2 | LIBS TECHNIQUE}

\section{1 | Basic principles}

Laser-induced breakdown spectroscopy (LIBS), also known as laser-induced plasma spectroscopy (LIPS) and laser spark spectroscopy (LSS), is a multi-element analytical technique based on atomic spectroscopy. LIBS can analyse solid, liquid and gaseous materials. In principle, the technique can quantify all elements with detection limits ranging from 0.3 to $100 \mu \mathrm{g} \mathrm{g}^{-1}$ (Noll, 2012). Because of its simple setup (Figure 1), LIBS can be used in a wide range of applications, including hazardous environments (e.g., radioactive and explosive), difficult to access and remote areas (e.g., deep ocean and Mars), and directly in the field (Lee, $\mathrm{Wu}, \mathrm{Lee}, \&$ Sneddon, 2004). A famous application of an LIBS system is its use in the Curiosity Rover to explore the soil composition on Mars (Grotzinger et al., 2012).

LIBS consists of focusing a high-energy laser pulse, with irradiance above $10^{9} \mathrm{~W} \mathrm{~cm}^{-2}$, on the sample surface to create a transient plasma and resolving the emission with a high-resolution spectrometer (usually less than $0.1 \mathrm{~nm}$ ). Plasma is a state of matter composed of atoms, ions, electrons and radiation in thermodynamic and electrostatic equilibrium, that is, temperature and chemical species distributions are in physical-chemical equilibrium (Cristoforetti et al., 2010). With such irradiance, the laser pulse can evaporate a small amount of the sample, whether solid, liquid or gaseous. The excited atoms, ions and electrons from the ablated sample form the plasma, which can reach a temperature of approximately $100,000 \mathrm{~K}$ at the moment of its ignition (Miziolek et al., 2006). During the laser pulse interaction and less than a microsecond after it, the plasma emits a broad range of radiation due to its high temperature. As the plasma cools, the background radiation attenuates, and the emission lines of the elements can be observed. The intensity of the emission lines depends on the delay between the laser pulse interaction and spectrum acquisition, and varies for each element. The longer the delay, the lower the background radiation and the emission line intensities. Thus, for each element, there is an optimum delay time, usually from 1 to $10 \mu \mathrm{s}$, which maximizes the signal-to-background ratio. Finding the optimum delay time for several elements is a difficult task and requires several combinations of parameters, which can be reduced by factorial design, as performed by Ferreira et al. (2009).

To identify and measure the emission lines, a set of collecting lenses and a fibre-optic cable direct the plasma emission to a spectrometer, which resolves the light spectrum. Because emission lines are specific to each element, the acquired spectrum reveals the sample fingerprint (i.e., its elemental composition), which can be used to identify soil types. With the emission line intensities, calibration models can be developed to estimate the element concentrations in soil samples (more details in the 'Quantitative Analysis' section).

\section{2 | Experimental setup}

A typical LIBS setup consists of a pulsed laser, a focusing lens, a sample holder, collecting lenses, a spectrometer, a time delay generator and a computer (Figure 1). Various laser systems (e.g., $\mathrm{CO}_{2}$ and Excimer) can be used, but LIBS mostly employs a neodymium-doped yttrium aluminium garnet (Nd:YAG) laser operating at its fundamental wavelength $(1,064 \mathrm{~nm})$ or one of its harmonics $(532,355$ or $266 \mathrm{~nm}$ ), typically in nanoseconds (Miziolek et al., 2006). Among laser parameters, the excitation wavelength is one of the most critical, as it affects the coupling between irradiation and the material surface.

For LIBS systems, the laser pulse duration can range from femtoseconds $\left(10^{-15} \mathrm{~s}\right)$ to microseconds $\left(10^{-6} \mathrm{~s}\right)$ and affects the resulting spectra. For femtosecond lasers, the pulse interacts so rapidly with the sample surface that it ceases before the plasma forms, in contrast to microsecond lasers, in which a considerable amount of pulse energy excites the species in the plasma. Nanosecond lasers lie in between femto- and microsecond lasers and were the most used in the reviewed applications.

Plasma emission is resolved by a spectrometer, usually a broad range polychromator and a charge-coupled device (CCD) or intensified charge-coupled device (ICCD) detector. Most polychromators operate from 200 to $1,000 \mathrm{~nm}$, where most of the emission lines of all elements are detectable. The resolution of the spectrometer defines the quality of a LIBS measurement, allowing consecutive emission lines to be separated without intricate processing, especially for 
spectra obtained from complex matrices, such as soil samples.

The delay generator is a fundamental component of the LIBS setup needed to synchronize the laser and the spectrometer with electrical pulses from nanoseconds to milliseconds. Fine-tuning of delay time allows optimization of the signal-to-noise/background ratio, which in turn enhances quantitative analysis.

A sample holder is an optional element of the system because LIBS can be applied directly to the sample, even in its original location. However, a sample holder is recommended to facilitate adjustment of the optics because all samples can be reliably placed at the same distance relative to the laser focus. For in situ analysis or samples with an irregular surface, an autofocus system may be required for optical adjustment.

Optimizing system parameters can considerably improve LIBS signal quality. In typical LIBS systems, four parameters require adjustment, namely: (a) laser pulse wavelength, (b) laser pulse energy, (c) delay time and (d) gate window. Depending on the application, the parameters can be optimized for each element separately or together. For soil analysis, LIBS usually includes the determination of several elements, including nutrients and toxic elements in a single measurement. Numerous combinations of parameters are possible, which require the evaluation of several experiments. Otherwise, this type of analysis becomes unfeasible in practice. To minimize the number of experiments, a factorial design (Deming, Palasota, \& Palasota, 1991) may be used, as was performed by Ferreira et al. (2009). This method allows maximizing the emission line intensities of several elements relative to the background with a feasible number of experiments.

One way to improve the quality of the LIBS signal is the use of a second pulse. Whereas the first pulse ablates a fraction of the sample surface and creates a plasma, the second re-excites the plasma to enhance the signal-to-noise ratio (Cremers \& Radziemski, 2013). This type of system is known as double-pulse LIBS (DP-LIBS) and may employ a single laser that shoots two consecutive pulses or two lasers shooting independent pulses. In either case, the inter-pulse delay must be short enough (usually a few microseconds) to re-excite the plasma during its lifetime (Cremers \& Radziemski, 2013). With two distinct lasers, a few configurations are possible: both pulses can be parallel or orthogonal at the same or different wavelengths. This setup requires several adjustments other than that for typical LIBS systems, such as the inter-pulse delay, the wavelength of both pulses and the optical configuration.

Recent advances in LIBS system setup have been proposed to improve the repeatability and sensitivity of the technique, as reviewed by Zorov et al. (2015): resonance laser excitation, spark discharge excitation, microwave excitation, plasma confinement in an external magnetic field, and plasma confinement in a microchamber. If a given element is not detected in soil sample analyses by the LIBS system used, such experimental configurations may be considered.

\section{3 | Qualitative analysis}

LIBS is an analytical tool widely used for qualitative analysis (Cremers \& Radziemski, 2013) because its fundamental principle relies on the identification of the emissions of the various chemical species in the plasma emission spectrum. For soil analysis, qualitative LIBS analyses are of great importance because they allow identification of not only the constituent elements but also the type of sample. This type of analysis can be used to discriminate between sets of samples, for example different types of soils, rocks and minerals (Zorov et al., 2015). The use of LIBS for qualitative analyses can also be very useful in environmental monitoring, especially for the detection of toxic elements in soils (Barbafieri, Pini, Ciucci, \& Tassi, 2011; Capitelli et al., 2002; Eppler, Cremers, Hickmott, Ferris, \& Koskelo, 1996; HilbkKortenbruck, Noll, Wintjens, Falk, \& Becker, 2001; Santos et al., 2009; Yamamoto, Cremers, Ferris, \& Foster, 1996).

A LIBS spectrum consists of numerous emission lines, each relating to a given chemical species, with excited electrons emitting photons at specific wavelengths, thus allowing identification of the elemental composition of the sample. Such identification is usually achieved by comparing the spectral peaks (such as those in Figure 2) with emission lines of established databases, such as NIST (Kramida et al., 2018) and Kurucz (Smith, Heise, Esmond, \& Kurucz, 1995). However, this is not an easy task, because samples

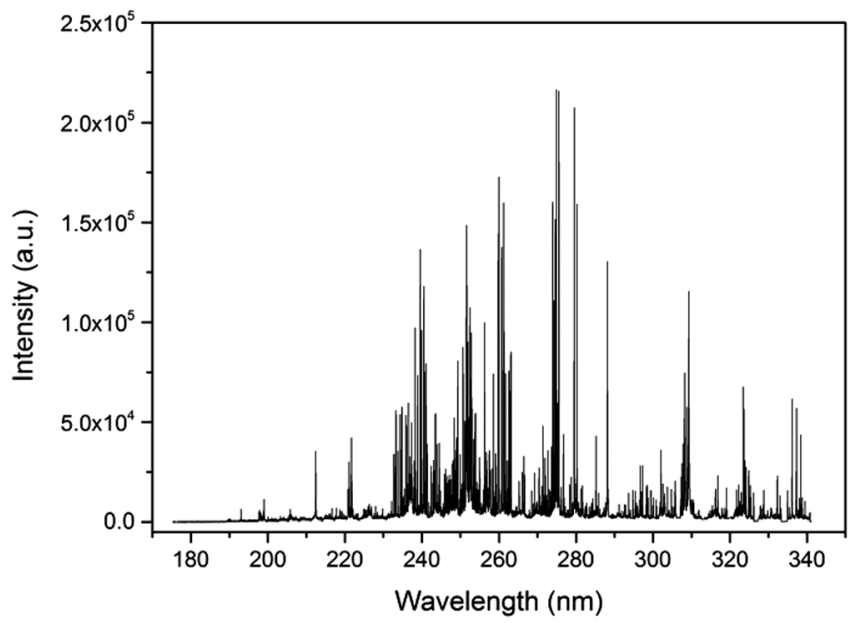

F I G U R E 2 Laser-induced breakdown spectroscopy (LIBS) spectrum of a soil sample containing emission lines of various chemical elements in different ionization states 
such as soils are generally heterogeneous and composed of several elements, thus leading to several emission lines in the spectrum (as in Figure 2). In addition, the position of the peaks may drift slightly (a shift of $0.05 \mathrm{~nm}$ is common) due to time and environmental conditions, making it even more difficult to identify the emission lines. Thus, such issues can result in misleading elemental identifications, but can be avoided or minimized with prior knowledge of the composition of the sample of interest. In qualitative analyses, the following factors should also be considered (Zorov et al., 2015):

- Presence of spectral lines at various stages of ionization, which depend on the experimental conditions. Generally, atomic and first ionization lines are observed in ambient air, whereas those of higher ionization levels (up to the fifth order) can be observed in a vacuum.

- The relative intensities of the spectral lines of each element extracted from the databases. Emission lines with the highest values of relative intensity are more likely to be found in the spectrum. Typically, these lines are persistent.

- Presence of multiplets. If a transition is observed in the spectrum, the other multiplets must also be detected.

- Identification of at least three emissions for a given chemical element to prove its existence in the sample.

Several studies (Refs. 91-99 as cited in Zorov et al., 2015) have developed techniques for improving qualitative analysis. Among these works, two methods deserve attention. The first considers the crustal abundance of elements (Ref. 95 as cited in Zorov et al., 2015). The second compares the experimental spectrum to synthetic spectra generated on the basis of the physical properties of the plasma and parameters of the emission lines (Refs. 99 and 100 as cited in Zorov et al., 2015). In the first method, the rank of a line for the element $n$, defined as MP metrics, is calculated by the expression:

$$
M P=\frac{c_{n}}{\left|\lambda_{m}-\lambda_{\text {theor }}\right|}
$$

where $c_{n}$ is the abundance of $n$ in the earth crust, and $\lambda_{m}$ and $\lambda_{\text {theor }}$ are the wavelengths of the observed peak and the transition line of $n$ in the database, respectively. This calculation must be done for the possible elements near the peak. The line is correctly identified if the $M P$ value of a certain element is three times greater than the value of other elements.

The second method generates synthetic spectra based on plasma physical properties, such as temperature and electron density at local thermodynamic equilibrium, and physical parameters of the emission lines of possible elements, such as energies of the fundamental and excited levels, Einstein coefficients and excited level degeneracy (Refs. 99 and 100 as cited in Zorov et al., 2015). Spectra are generated with various combinations of elemental composition and plasma parameters and are compared via Pearson correlation coefficient. The selection of the spectrum with the highest coefficient thus allows identification of the emission lines. This method was able to accurately identify more than 40 emissions between $393.34-413.04 \mathrm{~nm}$ in soil samples (Ref. 99 and 100 as cited in Zorov et al., 2015), which is a promising alternative to qualitative analyses.

\subsection{Quantitative analysis}

Quantitative analysis using LIBS is possible because the elemental abundances in the sample are proportional to their line intensities from the plasma emission. More strictly, quantitative analysis by LIBS is based on two assumptions: (a) the contents of an element in the plasma and in the sample are proportional, and (b) the emission line intensity of the species (either atom or ion) of an element in the LIBS spectrum is proportional to the concentration of the corresponding element in the plasma. Even though these assumptions may be satisfied, line intensity also depends on several interrelated factors, such as: laser pulse properties (wavelength, energy and duration), material characteristics (composition, optical transmissivity, reflectance, thermal conductivity and surface morphology), laser-material coupling, and environmental conditions (gas composition and pressure) in which the material is ablated (Harmon et al., 2013). The mechanisms involved in plasma formation are non-linear and are the result of the combination of these factors. Therefore, the assumptions cannot be satisfied a priori and rely on the criteria that the plasma must be (a) optically thin and (b) in local thermodynamic equilibrium. The first criterion implies that no electrons absorb the photons travelling to the collecting lenses. When an excited electron decays to a lower energy level in an atomic or ionic species, it emits a photon with a specific wavelength that can only be absorbed by a free electron or another that is in the lower level of the same species. Considering all of the directions in which a photon can be emitted, the probability is low of it being absorbed by an electron in its path to the collection lenses. However, denser plasmas or samples of a high concentration of a particular element may have a high probability. The second criterion requires that all atoms, ions and free electrons be at local thermodynamic equilibrium in the plasma (i.e., their temperature distribution must be characterized by the same mean) (Cristoforetti et al., 2010). A thorough plasma study may be required for quantitative analyses, as pointed out by Cristoforetti et al. (2010). 
Ideally, the intensity of an emission line is proportional to the abundance of the respective element in the sample. However, laser pulse properties, material characteristics, laser-material coupling and environmental conditions can still affect the line intensities. Even if all factors are controlled, the plasma formation, and consequently the line intensities, can still differ for consecutive laser pulses because of matrix effects. Every interaction between a laser pulse and sample surface results in a unique plasma because ablation and plasma formation depend on non-linear mechanisms. Thus, even the slightest variation in pulse power or duration may lead to a distinct plasma. Distinct matrices (e.g., soil and alloy) may considerably affect the line intensity of an element, such as phosphorus (P) and iron (Fe). To circumvent matrix effects, several approaches have been proposed including: (a) laser pulse averaging, (b) sample homogenization, (c) area normalization, and (d) the use of internal or external standards (Harmon et al., 2013). Laser pulse averaging aims to reduce the influence of laser pulse oscillation and sample surface heterogeneity. The second and third approaches help minimize the variation of line intensities caused by the sample heterogeneity. The use of an internal standard helps to correct the variations in ablation and plasma formation, whereas external standards are used to calibrate curves for the samples of interest.

Effectively measuring the concentration of an element in a sample may require several data treatments due to the complexity of an LIBS spectrum (see example in Figure 2), which depend on the sample type and the LIBS system. The usual data treatments for LIBS spectra are: (a) offset removal and baseline correction, (b) spectral normalization, (c) line intensity calculation and (d) calibration method development.

Baseline offset, commonly found in spectroscopy, is a constant signal independent of the samples analysed and is derived from system electronics, optics and experimental conditions. In general, the offset is the minimum value of a spectrum. In LIBS systems, a blank measurement (i.e., without sample) provides the best estimate for the offset. Such a procedure should be performed regularly to correct oscillations of laser properties, spectrometer parameters and environmental conditions during measurements.

The baseline is a recurring problem in LIBS spectra because of the strong residual background emission, mainly found in systems that cannot properly adjust the delay time, such as those with fixed parameters or configured for the simultaneous analysis of several elements. The baseline can be corrected either locally for just a couple of peaks or for a broad spectral range. In the first case, the most common approach is to fit a straight line to the points on the sides of one or more peaks, a method that is known as "correction of three points" (Dawson, Snook, \& Price, 1993). In the second case, an algorithm (e.g., Liland, 2015; Liland, Almøy, \& Mevik, 2010; Liland \& Mevik, 2011) fits a smooth curve to the baseline points that are in regions free of well-defined peaks. The first method is suitable for just a couple of peaks in a narrow region, whereas the second is suitable for a wide spectral range containing several interfered peaks. For element quantification, the first method is preferable. On the other hand, the second method is more appropriate for qualitative analyses, such as sample classification, and for the assessment of sample properties, such as soil $\mathrm{pH}$, in which the elements related to the property are not known.

Several normalization methods can be used to reduce matrix effects in LIBS, as was reviewed by Zorov, Gorbatenko, Labutin, and Popov (2010). Among these methods, background normalization and the use of an internal reference are the most used. Because line intensities are related to the plasma formation, and the plasma formation is, in turn, related to the laser-material interaction, all emission lines may be similarly affected by the plasma formation. Therefore, normalizing the line intensities by the area under the spectrum may minimize the effects of the plasma formation. Although helpful, background normalization may prove to be unsatisfactory for accurate and precise measurement using LIBS (Zorov et al., 2010). A better strategy for spectral normalization is the use of an internal reference. If an element is used as an internal reference in a sample set, then its emission line intensities can be used to normalize the emission lines of other elements. This solution underlies the fact that the matrix effects affect all emission lines similarly. For steel alloys, in which Fe concentration does not vary, $\mathrm{Fe}$ lines may be used to normalize the lines of other elements, such as P. Nevertheless, no element in soil has a constant concentration that can be used as an internal standard unless all samples are similar (i.e., the same type and with similar texture) or are mixed with a rare element (e.g., yttrium) at a given concentration. Although useful, adding a rare element to soil samples may be unfeasible for practical reasons (e.g., price and excess sample preparation).

The correct way to normalize the intensity of a line is by using the Boltzmann equation (e.g., Zorov et al., 2015). According to this equation, the ratio between the lines of two elements depends not only on the ratio of the concentration between the elements but also on the plasma temperature. Dependence on plasma temperature can be eliminated if the upper-level energy of the lines is very close. Thus, the concentration of an element can be determined by the internal reference concentration and the ratio between the lines of the two elements. Besides dependence on the local thermodynamic equilibrium, this process is impractical because it requires finding appropriate lines of the internal reference for each line to be normalized. To find appropriate lines for normalization, Zorov et al. (2015) proposed using the 
correlation coefficient between a line intensity of the element of interest and the emission signal intensity of each spectral point. The criterion for applying this method requires the following conditions: (a) high correlation coefficient with the line of the element of interest, (b) comparable intensities between the lines and (c) little variation in the concentration of an internal reference among samples. According to the authors, an advantage of such a criterion is that the method does not require local thermodynamic equilibrium. Using this procedure, Zorov et al. (2015) improved lead $(\mathrm{Pb})$ and molybdenum $(\mathrm{Mb})$ determination in soil samples.

In spectroscopy, the intensity of an emission line can be evaluated by the corresponding peak height or area. The peak area can be calculated numerically or by the area under the distribution estimated by deconvolution, a method that fits a suitable distribution, usually Gaussian, Lorentzian or Voigt (a mixture of Gaussian and Lorentzian) for the emission lines in an LIBS spectrum. Either the height or the numerical area can only be properly evaluated without interference from other peaks. In most cases, deconvolution is recommended because it best estimates a line intensity and can separate overlapped peaks.

The choice of emission lines is also crucial in LIBS analysis. In the range of 190 to $1,000 \mathrm{~nm}$, several emission lines of an element may be found in atomic emission databases, such as NIST (Kramida et al., 2018) and Kurucz (Smith et al., 1995). However, just a few emission lines may be useful for LIBS analysis, because of their transition probabilities, expected line intensities, and the state (atomic or ionic) they originated from. For example, from $100 \mathrm{C}$ emission lines in the NIST database, only a few may be found in the LIBS spectrum of a soil sample, because most of those lines have low transition probability or are from ions C II, C III and C IV. (The notation, element $\mathrm{E}$ followed by a capitalized Roman numeral, indicates the $\mathrm{E}$ ionization level in atomic emission databases (Kramida et al., 2018; Smith et al., 1995); for instance, C I is the atomic $\mathrm{C}, \mathrm{C}$ II is the first $\mathrm{C}$ ionization and $\mathrm{C} \mathrm{III} \mathrm{is} \mathrm{the} \mathrm{sec-}$ ond $\mathrm{C}$ ionization.) First ionized $\mathrm{C}$ is unlikely to be found in the plasma, due to the high ionization energy $(11.26 \mathrm{eV})$ required to remove an electron from atomic $\mathrm{C}$ compared to the energy of $8.62 \mathrm{eV}$ to create a plasma at 100,000 K. (In plasma theory, the plasma energy is $k_{B} T$, where $k_{B}$ is the Boltzmann constant and $T$ is the temperature; therefore, $1 \mathrm{eV}$ is equivalent to 11,604.5 K.) Metals such as aluminium ( $\mathrm{Al})$, on the other hand, are likely to be found in the first ionized state, due to their low ionization energy (e.g., first $\mathrm{Al}$ ionization requires $5.99 \mathrm{eV}$ ). Another problem commonly found in LIBS analysis is the selfabsorption of emission lines. When the transition probability of an emission line is high, the corresponding photon is likely to be absorbed by an electron in the plasma. At low concentrations, the probability is low, but as the concentration increases, the probability also increases. Therefore, the intensity of such a line does not vary linearly with the concentration of the corresponding element and should be avoided for calibration models.

Univariate or multivariate and linear or non-linear models can be used to calibrate LIBS for soil analysis (e.g., Ferreira et al., 2011; Ferreira, Milori, Ferreira, Da Silva, \& Martin-Neto, 2008; Nicolodelli et al., 2014; Segnini et al., 2014). The univariate and linear model may be suitable in most cases. However, the intensity of a single emission line may not reflect the variation of an element concentration, because of self-absorption, weak signal or interference by lines of other elements. To overcome such problems or to improve LIBS accuracy, multivariate models may be used, such as partial least square regression (PLSR), support vector machine (SVM) and artificial neural networks (Zhang et al., 2015). Such models can also be calibrated with a large spectral region rather than emission line intensities. To calibrate models, a given sample set (namely training set) is used to determine the weights of the spectral points according to the concentration of the element of interest. The weighting process is usually performed automatically and considers spectral points with strong correlations with the concentration of elements of interest. The use of such models considering a wide spectral region is useful when LIBS spectra are too intricate for the emission lines to be properly separated. Nevertheless, multivariate models may overfit the training set (i.e., the models are likely to have a poor predictive performance with samples not belonging to the training set). Therefore, linear models considering emission line intensities are preferable to non-linear multivariate models that use large spectral regions. More details on chemometrics applied to LIBS analysis can be found in the reviews by Hahn and Omenetto (2012) and Zhang et al. (2015).

In addition to the univariate and multivariate models, the concentration of an element can be obtained by a theoretical model, known as the calibration-free method (Ciucci et al., 1999), without the need for a calibration curve. In this method, the concentration of an element $\alpha$ is given by the total emitter density, $N_{\alpha}$, as:

$$
C_{\alpha}=N_{\alpha} A_{\alpha} \chi_{p}
$$

where $A_{\alpha}$ is the atomic mass of $\alpha$ and $\chi_{p}$ is plasma normalization constant defined by:

$$
\chi_{p}=\left(\sum_{i} N_{i} A_{i}\right)^{-1}
$$

or can be obtained by Equation (2) if the concentration of an element is known. The term $N_{\alpha}$ is commonly approximated by the sum of $N_{\alpha I}$ and $N_{\alpha I I}$, the emitter density of $\alpha I$ and $\alpha I I$. The emitter density of species $s$ (e.g., $\alpha I$ or $\alpha I I$ ), $N_{s}$, can be 
obtained by the Boltzmann equation (Gornushkin, Merk, Tognoni, \& Panne, 2010):

$$
I_{s}=\frac{N_{s} h c}{4 \pi U_{s}(T)} \frac{g_{k}^{s} A_{k i}^{s}}{F(\lambda)} e^{-\frac{E_{k}^{s}}{K_{B} T}}
$$

where $I_{s}$ is the intensity of an $s$ emission line, $F(\lambda)$ is the spectrometer function, $g_{k}^{s}$ is the level $k$ degeneracy of $s, A_{k i}^{s}$ is the Einstein coefficient of $s$ for spontaneous emission from level $k$ to $i, h$ is the Planck constant, $c$ is the speed of light, $K_{B}$ is the Boltzmann constant, $T$ is the plasma temperature, and $U_{s}(T)$ is the $s$ partition function at temperature $T$. The main advantage of this method is that the matrix effects are minimized because plasma parameters are taken into account (Ciucci et al., 1999).

\section{5 | Sample preparation}

Soils are among the most complex matrices for LIBS analysis due to the heterogeneity of constituents and particle size (Jantzi et al., 2016). For instance, the spectra of two consecutive LIBS measurements of a soil sample may differ even if all conditions are the same. This problem can be aggravated if intact soil samples are analysed (Bricklemyer, Brown, Barefield, \& Clegg, 2011; Bricklemyer, Brown, Turk, \& Clegg, 2013). In this case, the variation among spectra of each sample can be too great. To compensate for this issue, the analyst can average numerous shots (e.g., 100) or normalize each spectrum. The LIBS analysis can also be performed on powdered soil samples, which were ground and homogenized either manually with mortar and pestle or mechanically. Thus, the resulting spectra vary less than those obtained for intact samples, but the preparation requires extra minutes per sample. Ground and homogenized soil samples can also be pelletized to ameliorate surface roughness. Although sample preparation time increases ( $\sim 5 \mathrm{~min} / \mathrm{sample}$ ), the resulting spectra vary the least for pelletized samples in relation to the other types of soil sample preparations, because heterogeneity and surface roughness are minimized. For direct in-field measurements in which time is crucial and soil sample preparation is not practical, averaging a large number of measurements on the sample surface may reduce errors and be better suited for this condition. For all types of samples, including soil, detailed sample preparation can be found in the work of Jantzi et al. (2016).

\section{3 | SELECTED SOIL CHEMICAL AND PHYSICAL PROPERTIES}

Soil chemical properties, such as $\mathrm{pH}$, cation exchange capacity and salinity, are related to many important processes that occur in soils, including mineralization, solubilization, the decomposition rate of SOM, and immobilization of minerals and nutrients because of biological activity. The analysis of chemical properties usually requires reagents and a long laborious process, which may affect the results. Because chemical properties are indirectly related to the elemental composition, they can be estimated by LIBS in conjunction with multivariate methods. For instance, soil salinity is measured by the quantification of sodium $\left(\mathrm{Na}^{+}\right)$, potassium $\left(\mathrm{K}^{+}\right)$, calcium $\left(\mathrm{Ca}^{2+}\right)$, magnesium $\left(\mathrm{Mg}^{2+}\right)$ and chloride $\left(\mathrm{Cl}^{-}\right)$ions and their concentrations may be directly determined by LIBS. Therefore, a combination of emission lines of such elements may provide a useful estimation for soil salinity. In this section, we will present the use of LIBS to estimate soil $\mathrm{pH}$, the humification degree of SOM and soil texture.

\subsection{Soil pH}

Acid-base equilibrium in soil, evaluated by $\mathrm{pH}$, is involved in many biochemical processes and also affects other soil physical, chemical and biological properties (McLean, 1982). Even soil productivity is affected by such an equilibrium. Usually, soil $\mathrm{pH}$ is determined in slurries of water and soil (McLean, 1982) or addition of a $\mathrm{CaCl}_{2}$ solution to minimize problems with suspended particles and variable salt content (Schofield \& Taylor, 1955). Nonetheless, sample preparation differs for each type of soil, which demands a previous characterization and further analytical process to determine soil $\mathrm{pH}$. LIBS offers an alternative method to estimate soil $\mathrm{pH}$ because many elements are related to soil acidity or alkalinity. For instance, $\mathrm{Ca}$ is directly related to soil $\mathrm{pH}$, because the presence of $\mathrm{Ca}$ indicates soil alkalinity, and soil liming is a common practice to correct soil acidity. In addition, exchangeable $\mathrm{Al}$ dominates acid soils and may precipitate to form aluminium hydroxide $\left(\mathrm{Al}(\mathrm{OH})_{3}\right)$ with liming. Thus, $\mathrm{Ca}$ and $\mathrm{Al}$ together with $\mathrm{H}$ and $\mathrm{O}$ lines were considered in developing a multivariate calibration model for soil $\mathrm{pH}$.

The following study considered 60 samples covering a broad range of Brazilian soils with $\mathrm{pH}$ between 4.0 and 6.3 and standard deviation of less than $0.2 \mathrm{pH}$ units (Ferreira et al., 2015). The samples were cryogenically ground with liquid $\mathrm{N}$ to reduce heterogeneity and were pelletized with a 10 -ton press for the LIBS measurements. The pelletized soils were subjected to an LIBS system using a Nd:YAG laser operating at $532 \mathrm{~nm}$ with a pulse of $115 \mathrm{~mJ}$ energy, $4.4 \mathrm{~ns}$ pulse duration and $10 \mathrm{~Hz}$ repetition rate. The LIBS spectra were acquired by an Echelle spectrograph with a focal length of $195 \mathrm{~mm}, \mathrm{~F} / 7$, from 200 to $975 \mathrm{~nm}$, coupled with an ICCD detector. The delay between the laser pulse and spectra acquisition was $400 \mathrm{~ns}$ and the spectrograph integration time gate was $25 \mu$ s. The calibration model was developed using 
T A B LE 1 Comparison between the reference and predicted $\mathrm{pH}$ values

\begin{tabular}{|lll|}
$\begin{array}{l}\text { pH reference } \\
\text { value }\end{array}$ & $\begin{array}{l}\text { pH predicted by LIBS and } \\
\text { PLSR method }\end{array}$ & $\begin{array}{l}\text { Absolute } \\
\text { error }\end{array}$ \\
\hline 6.2 & 4.9 & 1.3 \\
\hline 5.4 & 5.0 & 0.6 \\
\hline 4.5 & 4.4 & 0.1 \\
\hline 5.4 & 5.3 & 0.1 \\
\hline 5.0 & 5.1 & 0.1 \\
\hline 4.5 & 4.5 & 0.0 \\
\hline 5.6 & 5.6 & 0.0 \\
\hline 4.1 & 4.4 & 0.3 \\
\hline 4.8 & 4.7 & 0.1 \\
\hline 5.9 & 5.7 & 0.2 \\
\hline
\end{tabular}

Reprinted with permission from the work of Ferreira et al. (2015).

the PLSR method and with narrow regions around the emission lines of Ca II at 373.69, 393.33 and $396.82 \mathrm{~nm}, \mathrm{Al}$ I at 394.42 and $396.15 \mathrm{~nm}, \mathrm{H} \mathrm{I}$ at $656.11 \mathrm{~nm}$ and O II at 655.46, 656.51 and $657.11 \mathrm{~nm}$ as input variables. Calibrated with 50 samples (training set), the model estimated the soil $\mathrm{pH}$ of the remaining 10 samples (test set) with $R=0.86$ and a root mean error of prediction of $0.4 \mathrm{pH}$ units (Table 1) considered a satisfactory uncertainty compared to the $0.2 \mathrm{pH}$ units of the reference technique.

\section{2 | Humification degree of soil organic matter (SOM)}

Organic matter is crucial for the health of soil because it provides a source of nutrients to plants and microorganisms, promotes good physical soil structure and minimizes erosion, and constitutes one of the most important $\mathrm{C}$ reservoirs on Earth. In particular, SOM represents the largest terrestrial $\mathrm{C}$ pool and its maintenance depends directly on soil management practices in managed systems (Falkowski, 2000). However, understanding which soil management practice is suitable for each type of soil is not an easy task and requires several decades of experiments and analyses to determine not only the content of $\mathrm{C}$ in soils but also its stability.

Carbon stability cannot be measured directly but is to a certain extent related to the chemical structure of organic matter, which can be assessed by the humification degree, an index associated with the complexity of the chemical structure. More specifically, the chemical pathways of decomposition and humification of SOM enhance the concentration of recalcitrant chemical structures, such as complex conjugated aromatic rings. To evaluate such structures, a chemical fractionation process is typically performed to separate the humic substances: humin, humic and fulvic acids. This is a complex, laborious and long process (generally requiring 15 days). The structure of these substances can be assessed by nuclear magnetic resonance (NMR) and electron paramagnetic resonance (EPR). However, these techniques cannot be used for soil rich in $\mathrm{Fe}$, such as Brazilian soils. For this reason, fluorescence spectroscopy is a valuable alternative to evaluate the structure of humic substances, despite the need for the separation step. Recently, Milori et al. (2006) demonstrated that the fluorescence area of bulk soil was proportional to the fluorescence index of the separated humic substances and created the index $H_{L I F S}$, which corresponds to the ratio between the fluorescence area and the $\mathrm{C}$ concentration. Although this simplified the process of analysing the humification degree of SOM, it still required the elemental analyser and fluorescence spectroscopy to evaluate $H_{\text {LIFS }}$. Because the humification degree depends on the composition of SOM, LIBS can be used to estimate this index based on the stoichiometric relation between elements in the soil samples. Ferreira et al. (2014) evaluated the humification degree of 56 soil samples, classified as sandy Yellow Red Argisol, collected from 0 to $80-\mathrm{cm}$ soil depth of two sugar cane fields, with and without burning. The samples were ground with a mortar and pestle and subsamples were then used to determine $\mathrm{C}$ using an elemental analyser, while the remainder was pelletized with an 8 -ton press. The pellets were then analysed by fluorescence spectroscopy (non-destructive technique) first, followed by LIBS.

The system used for fluorescence spectroscopy consisted of a continuous wave laser operating at $405 \mathrm{~nm}$ to excite the organic substances and a spectrometer to acquire the fluorescence spectra $(420$ to $800 \mathrm{~nm})$. The integration time, the number of measurements for averaging and the boxcar were $400 \mathrm{~ms}, 3$, and 3, respectively. The LIBS system consisted of a Nd:YAG laser, operating at $1064 \mathrm{~nm}$, pulse energy of $50 \mathrm{~mJ}$, a pulse duration of $8 \mathrm{~ns}$ and seven spectrometers with a resolution of $0.1 \mathrm{~nm}$ covering the range of 188 to $980 \mathrm{~nm}$. The delay time and the integration time were adjusted to $10 \mu \mathrm{s}$ and $2 \mathrm{~ms}$, respectively. For each sample, 60 measurements were acquired and each spectrum was normalized by the respective area in the range of 190.8 to $500.6 \mathrm{~nm}$. The region outside this range was disregarded for analysis. Normalization by area was used to reduce the spectral dispersion caused by the matrix effects (Cremers \& Radziemski, 2013). The normalized spectra were averaged for each sample.

To estimate the humification degree of SOM by LIBS, two models were developed: one for $\mathrm{C}$ concentration and another for the fluorescence area. The $\mathrm{C}$ concentration was estimated by the emission line at $193.03 \mathrm{~nm}$ corrected by the Al line at $193.56 \mathrm{~nm}$, as described in the 'Carbon' section in Part II of this review (Villas-Boas et al., 2019, this issue). Two steps were used for the fluorescence area: (a) selecting 

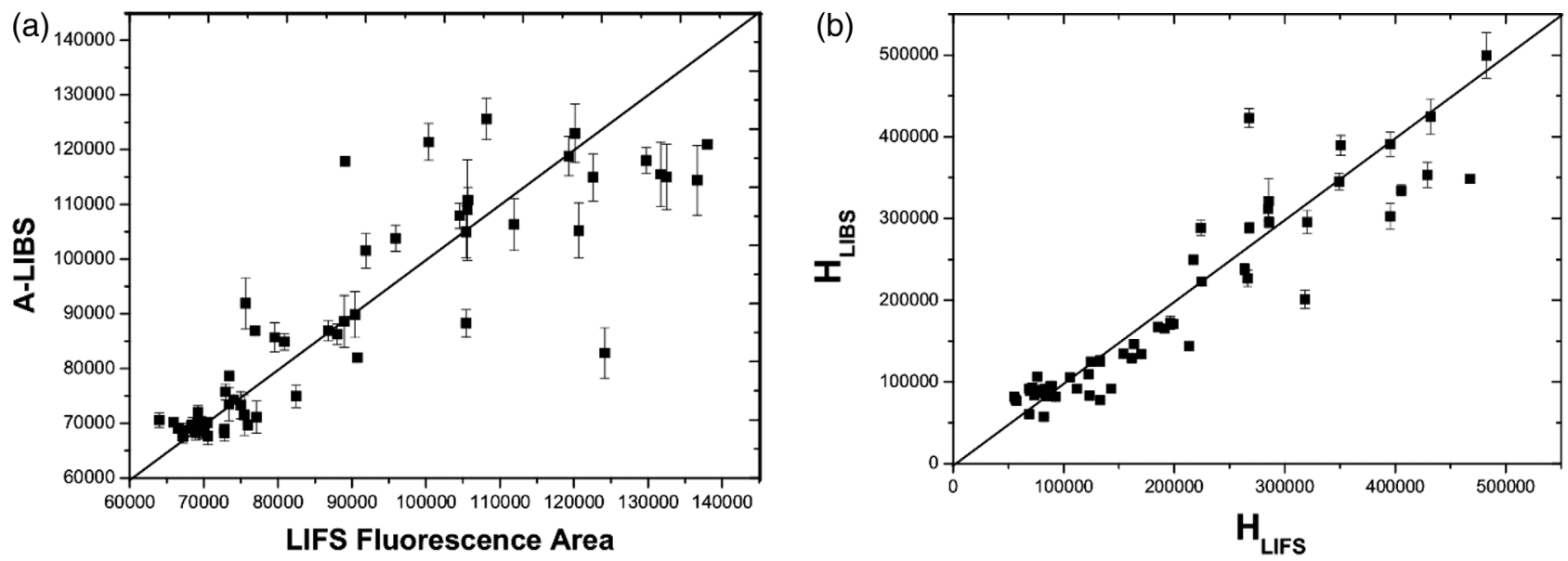

F I G URE 3 (a) Validation of the fluorescence area estimated by laser-induced breakdown spectroscopy (LIBS); (b) validation of the humification degree estimated by LIBS. Reprinted with permission from the work of Ferreira et al. (2014)

the emission lines and (b) developing the calibration model. To select the lines, the entire spectral region was correlated with the fluorescence area, which was considered as a function of each wavelength (independent variable). Only wavelengths showing linear, quadratic or exponential behaviour were considered in the calibration model. Thus, the selected lines were: Al I at 394.42, Al II at $198.96 \mathrm{~nm}$;g I at 285.22, Mg II at 279.55 and $280.26 \mathrm{~nm}$; and $\mathrm{Ca}$ I at 422.65, $\mathrm{Ca}$ II at 317.95, 393.38, 396.84 and $396.91 \mathrm{~nm}$. The calibration model used in this analysis was based on the k-nearest neighbour technique (k-NN) (Aha, Kibler, \& Albert, 1991), with $\mathrm{k}=5$, and was tested with a 10-fold cross-validation. (An $\mathrm{N}$-fold cross-validation is a procedure that splits samples into $N$ sets and tests the performance of a calibration model. The model is developed with $N-1$ sets and tested with the remaining set. The procedure is repeated until all sets are tested.) The results are shown in Figure 3a. A Pearson correlation coefficient of 0.87 and root average error of $33.5 \%$ was obtained for the fluorescence area estimated by LIBS (A-LIBS). Despite the scatter dispersion in Figure 3a, the humification degree estimated by LIBS $\left(H_{L I B S}\right)$ showed a high correlation coefficient (0.94) to the humification degree evaluated by LIFS $\left(H_{\text {LIFS }}\right)$ (Figure $\left.3 \mathrm{~b}\right)$. Therefore, $H_{\text {LIBS }}$ was to some extent corrected for $\mathrm{C}$ concentration also estimated by LIBS. In other words, the errors of one estimate partially compensated for the errors of the other.

The selection line analysis indicated that the fluorescence area was positively correlated with the emission lines of $\mathrm{Al}$ but negatively correlated with the emission lines of $\mathrm{Mg}$ and $\mathrm{Ca}$. Therefore, as organic matter becomes more humified, it tends to bind to $\mathrm{Al}$, while tending to lose $\mathrm{Mg}$ and $\mathrm{Ca}$, which probably belong to organic compounds composed mainly of aliphatic chains instead of complex aromatic rings. More information on humification degree of SOM can be found in the review by Senesi, Martin-Neto, Villas-Boas, Nicolodelli, and Milori (2018).

\section{3 | Physical properties: Soil texture}

Soil texture affects the susceptibility to erosion, nutrients and pollutant leaching, water-retention capacity, drainage and organic matter content. Traditionally, soil texture is determined by the pipette or hydrometer method, but both are laborious and require day-long pretreatment to disperse clay particles and aggregates. Other methods have been developed for the direct determination of soil particle size, for example, gamma-ray attenuation (Naime, Vaz, \& Macedo, 2001), X-ray attenuation, laser diffractometry, photometrical techniques and electroresistance particle counting (McCave \& Syvitski, 1991), but all of them require a dispersion step. Methods for indirect determination, such as visible and near infrared reflectance spectroscopy, have also been proposed (Chang, Laird, Mausbach, \& Hurburgh, 2001; Curcio, Ciraolo, D'Asaro, \& Minacapilli, 2013; Madari et al., 2006; Viscarra Rossel, Walvoort, McBratney, Janik, \& Skjemstad, 2006), but their accuracy depends on environmental conditions, such as temperature and humidity, when the samples are analysed.

In principle, soil texture cannot be determined by its elemental composition due to its relation to the size of particles which is a physical feature. However, because soils are formed by rock weathering, which is a combination of physical, chemical and biological processes, their mineral particles are derived from the source geology, which may ultimately define the size of particles. In other words, rocks do not break down evenly, even in the same weathering conditions, due to their crystalline structure. Therefore, the size of a particle is to a certain extent related to its origin, which in turn is related to its elemental composition. 
(a)

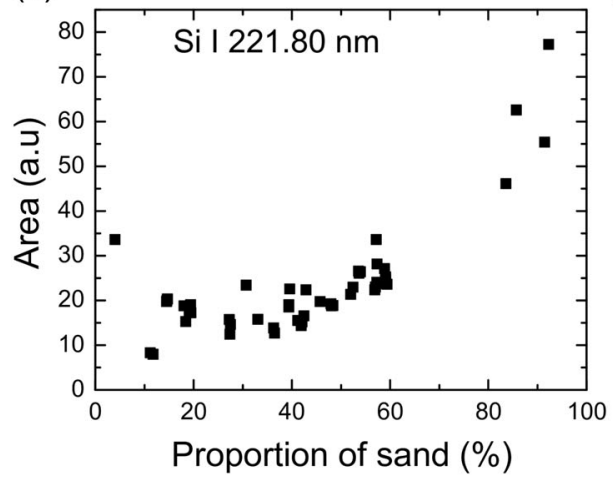

(b)

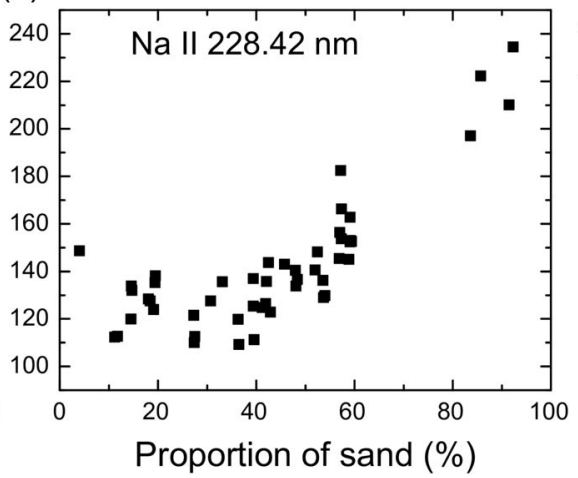

(c)

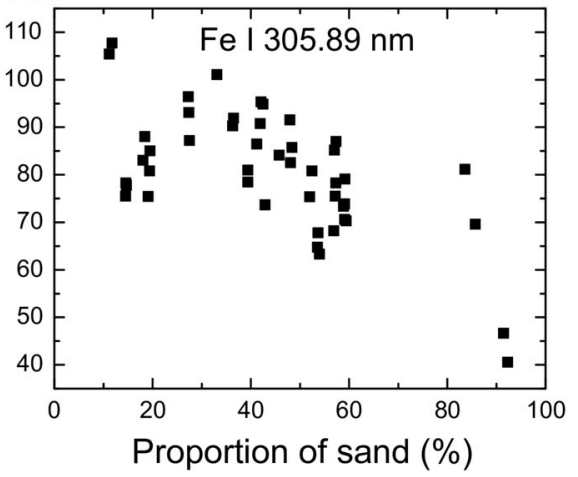

F I G URE 4 Scatterplot of the relation between Si I (a), Na II (b) and Fe I (c) emission lines and the proportion of sand. Reprinted with permission from the work of Villas-Boas et al. (2016)

This section covers how LIBS was used to estimate the percentage of sand, clay and silt of soil samples (Villas-Boas et al., 2016).

This study took into account the 60 soil samples presented in the 'Humification degree of SOM' section and measured with the same LIBS system. Each soil sample was measured 60 times. Instead of building calibration models with the proportions of sand $(\alpha)$, clay $(\beta)$ and silt $(\gamma)$, the variables were transformed as follows:

$$
\left\{\begin{array}{r}
\varepsilon=\alpha-\beta-\gamma \\
\phi=\beta-\gamma
\end{array}\right.
$$

Such a transformation allows reducing the uncertainty of estimating the three components because the uncertainty of a calibration model is inevitably higher than that of the reference method for each component individually. Thus, the uncertainty of estimating two variables is lower than that of estimating three. The calibration models were then developed considering the variables $\varepsilon$ and $\phi$, and the estimated relative proportions were recovered by:

$$
\left\{\begin{array}{r}
\alpha=\frac{1+\varepsilon}{2} \\
\beta=\frac{1+2 \phi-\varepsilon}{4} \\
\gamma=\frac{1-2 \phi-\varepsilon}{4}
\end{array}\right.
$$

To calibrate LIBS, two approaches were used: one considering the entire spectral range from 188 to $980 \mathrm{~nm}$, henceforth called model A, and another considering emission lines with high correlation with the proportions of sand, clay or silt, henceforth called model B. Both models were developed using PLSR. Prior to developing the models, the offset of each spectrum was subtracted and the samples were split into two sets: one for data treatment and another for model development. The first set contained 15 samples manually chosen with varying texture from sandy to clay soils.

The data treatment consisted of a baseline correction method and a peak-finding procedure. The method used for baseline correction was 4S Peak Filling (Liland, 2015), which had been successfully applied to other techniques, such as NMR and Raman spectroscopies (Liland et al., 2010; Liland \& Mevik, 2011). Using a genetic optimization algorithm (Mebane Jr \& Sekhon, 2011), the method was optimized with the first 15 samples and individually for each spectrometer region due to their intrinsic response functions. Later, the corrected spectrum was averaged for each sample and used in the peak-finding procedure, which consisted of correlating each wavelength with the soil texture and selecting emission lines with an absolute correlation coefficient higher than 0.8 for any of the textural proportions. According to this criterion, the emission lines were selected from the species: silicon (Si) I, Si II, titanium (Ti) I, Ti II, Fe I, Fe II, Na I, Na II, Ca I, Ca II, K I, Al I, cobalt (Co) II, Mg I, Mg II, V I, barium (Ba) I, Ba II, and beryllium (Be) I. The $4 \mathrm{~S}$ peak filling parameters optimized with the first set were used to develop model A with the test set. Analogously, model B was developed with the area of the selected emission lines determined by the deconvolution procedure ('Carbon' section in Part II of this review (VillasBoas et al., 2019, this issue)) with Lorentzian distribution for the peaks.

A leave-one-out cross-validation was applied to models $\mathrm{A}$ and $\mathrm{B}$, resulting in an average Pearson correlation coefficient of 0.89 and 0.9 , respectively, and an average RMSE of $6 \%$ for both models. Even though the accuracy of model B is equivalent to the accuracy of model A, model B should be more robust than model A in practice, because it is based on specific emission lines rather than a large spectral region, as 
T A B L E 2 Comparison between the classification using the pipette method and that using laser-induced breakdown spectroscopy (LIBS) coupled with model B

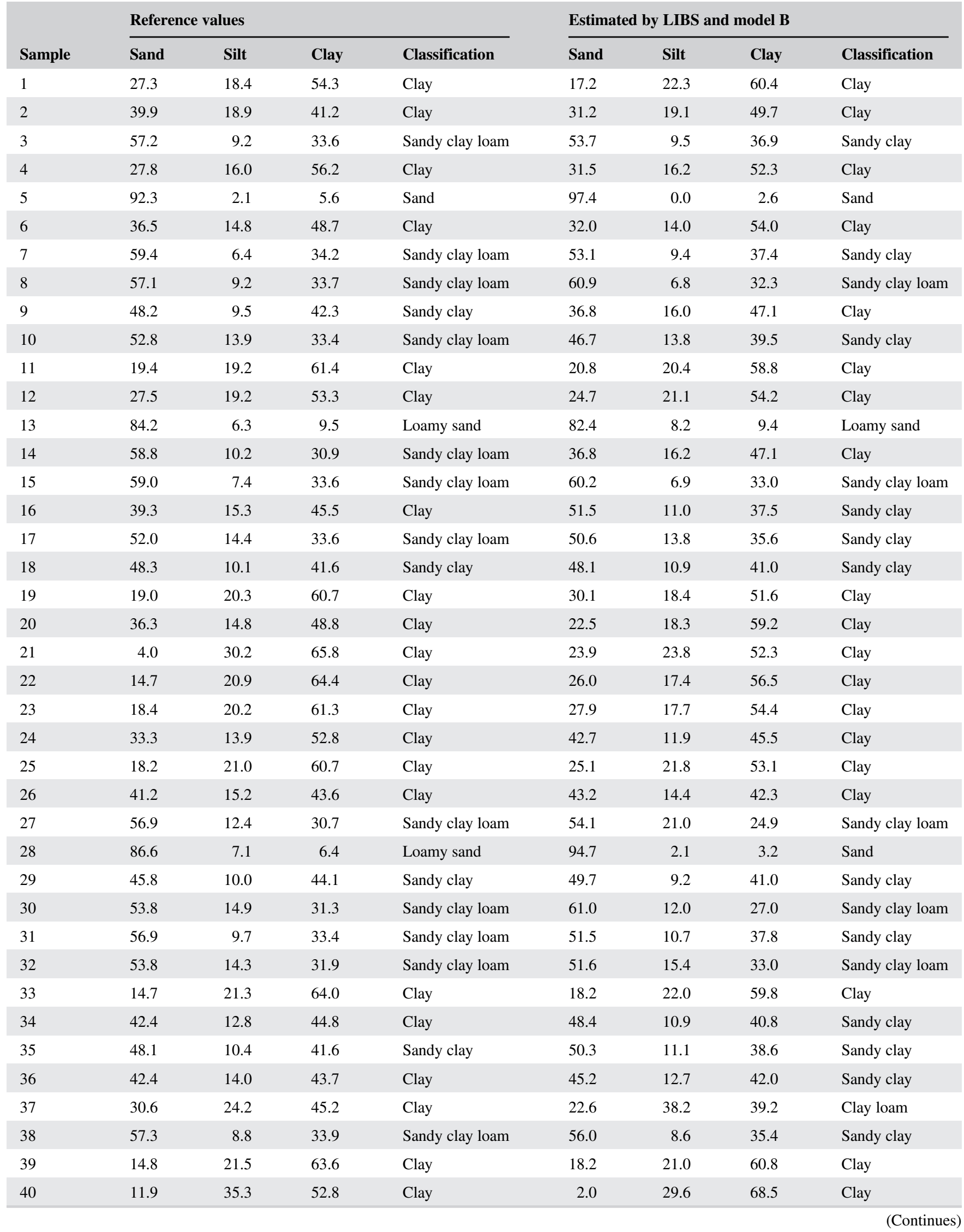


T A B L E 2 (Continued)

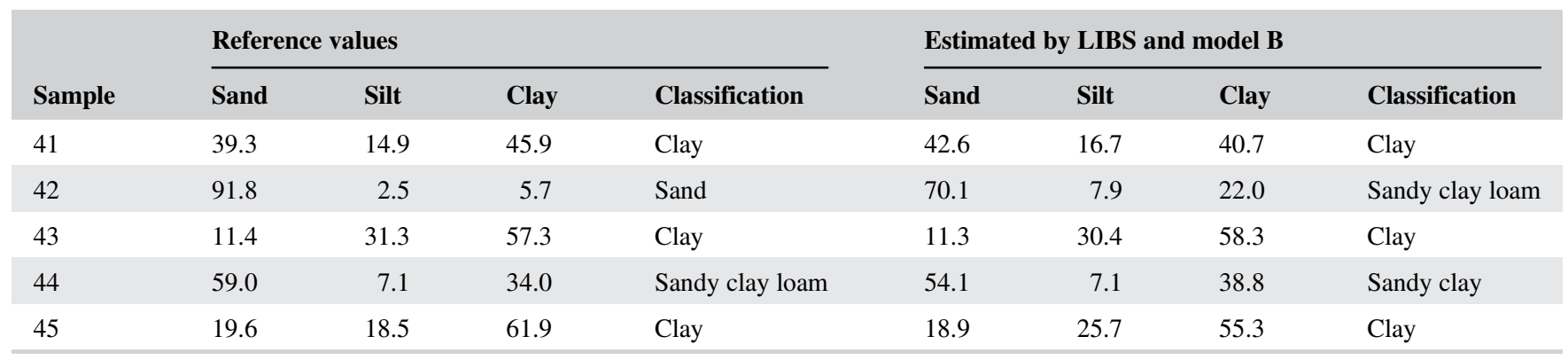

Reprinted with permission from the work of Villas-Boas et al. (2016).

in model A. Model A should be more susceptible to the matrix effects than model $\mathrm{B}$, because the former may have taken into account noise and background emission. The peak-finding procedure also indicated which elements are more abundant in clay, sand and silt. For instance, the lines $\mathrm{Si}$ I at $221.80 \mathrm{~nm}$ and $\mathrm{Na}$ II at $228.4 \mathrm{~nm}$ were positively correlated with the proportion of sand, whereas $\mathrm{Fe} \mathrm{I}$ at $305.89 \mathrm{~nm}$ was negatively correlated (Figure 4 ). The positive $\mathrm{Si}$ correlation with sand proportion was expected because most sand particles are derived from silica $\left(\mathrm{SiO}_{2}\right)$. In addition, the Na positive correlation may be related to the type of Brazilian rock (probably feldspar) from which the sand originated. Conversely, the observed negative Fe correlation may be a consequence of clay particles being rich in Fe oxides.

In conclusion, LIBS estimated the soil textural proportions with accuracy higher than 0.85 and uncertainty of $6 \%$ on average for both models. The uncertainty was reasonable compared to that of the pipette method (3\%), which means that LIBS could be used to scan large areas because it is a quick way to estimate the soil texture. According to the soil texture triangle (Saxton, Rawls, Romberger, \& Papendick, 1986), LIBS together with model B classified correctly most soil samples (Table 2), except for only two samples classified more than two classes distant. More recently, Knadel et al. (2017) used the LIBS procedure and associated model analysis developed by Villas-Boas et al. (2016), with excellent results for soil texture determination for Danish agricultural soils. Knadel et al. (2017) also compared LIBS to NIRS analysis (including soil C determination) and concluded that LIBS resulted in lower prediction errors for most properties than the well-established visNIRS method.

\section{4 | CONCLUDING REMARKS}

In this, the first of a two-part review, we covered LIBS fundamentals and recent LIBS studies in assessing soil chemical and physical properties. We showed that LIBS is a technique with great potential for soil chemical and physical characterization, due to its simple setup, low cost per sample analysis, simplified sample preparation and ability to detect a broad range of elements. Despite traditional LIBS use for soil elemental analysis, including C, nutrients and toxic elements, we demonstrated how to use LIBS to assess complex soil chemical and physical properties, such as soil $\mathrm{pH}$, humification degree of SOM and soil texture. Such a result is possible because of the relation between elemental composition and soil chemical and physical properties, for instance the relation of soil $\mathrm{pH}$ to $\mathrm{Ca}$ and $\mathrm{Al}$ concentrations.

The main advantage of using the LIBS technique for soil analysis is that we can evaluate various soil properties from only one measurement, not to mention the other intrinsic advantages, such as analysis speed, low cost per analysis and analysis without reagents. Although not as precise and accurate as reference techniques, LIBS can be very useful for screening large areas, thus allowing monitoring of various soil properties, as well as the relative abundance of nutrients and contaminants. The use of the technique for other properties, such as cation exchange capacity, salinity and water-retention curve, remains to be evaluated. In the second part of this review, we will discuss the LIBS applications to soil classification and soil elemental analysis, including soil $\mathrm{C}$, nutrients and toxic elements. We will cover the main LIBS advantages, limitations and challenges for soil elemental analysis.

\section{ACKNOWLEDGEMENTS}

This work was supported by CNPq (grants 312647/2018-2, 153993/2017-0 and 403405/2013-0) and FAPESP (grant 2013/07276-1).

\section{CONFLICT OF INTEREST}

The authors declare no conflict of interest. 


\section{DATA AVAILABILITY STATEMENT}

Data sharing is not applicable to this article as no new data were created or analysed in this study.

\section{ORCID}

\section{Paulino R. Villas-Boas (D) https://orcid.org/0000-0002-8583-} 3954

Marco A. Franco (1) https://orcid.org/0000-0002-2279-7722

\section{REFERENCES}

Aha, D. W., Kibler, D., \& Albert, M. K. (1991). Instance-based learning algorithms. Machine Learning, 6, 37-66.

Barbafieri, M., Pini, R., Ciucci, A., \& Tassi, E. (2011). Field assessment of $\mathrm{Pb}$ in contaminated soils and in leaf mustard (Brassica juncea): The LIBS technique. Chemistry and Ecology, 27, 161-169.

Borghi, E., Avanzi, J. C., Bortolon, L., Luchiari Junior, A., \& Bortolon, E. S. O. (2016). Adoption and use of precision agriculture in Brazil: Perception of growers and service dealership. Journal of Agricultural Science, 8, 89.

Bousquet, B., Sirven, J.-B., \& Canioni, L. (2007). Towards quantitative laser-induced breakdown spectroscopy analysis of soil samples. Spectrochimica Acta Part B, 62, 1582-1589.

Bray, R. H., \& Kurtz, L. T. (1945). Determination of total, organic and available form of phosphorus in soils. Soil Science, 59, 39-46.

Bricklemyer, R. S., Brown, D. J., Barefield, J. E., \& Clegg, S. M. (2011). Intact soil core total, inorganic, and organic carbon measurement using laser-induced breakdown spectroscopy. Soil Science Society of America Journal, 75, 1006.

Bricklemyer, R. S., Brown, D. J., Turk, P. J., \& Clegg, S. (2018). Comparing vis-NIRS, LIBS, and combined vis-NIRS-LIBS for intact soil core soil carbon measurement. Soil Science Society of America Journal, 82, 1482.

Bricklemyer, R. S., Brown, D. J., Turk, P. J., \& Clegg, S. M. (2013). Improved intact soil-core carbon determination applying regression shrinkage and variable selection techniques to complete spectrum laser-induced breakdown spectroscopy (LIBS). Applied Spectroscopy, 67, 1185-1199.

Capitelli, F., Colao, F., Provenzano, M. R., Fantoni, R., Brunetti, G., \& Senesi, N. (2002). Determination of heavy metals in soils by laser induced breakdown spectroscopy. Geoderma, 106, 45-62.

Chang, C.-W., Laird, D. A., Mausbach, M. J., \& Hurburgh, C. R. (2001). Near-infrared reflectance spectroscopy-principal components regression analyses of soil properties. Soil Science Society of America Journal, 65, 480.

Ciucci, A., Corsi, M., Palleschi, V., Rastelli, S., Salvetti, A., \& Tognoni, E. (1999). New procedure for quantitative elemental analysis by laser-induced plasma spectroscopy. Applied Spectroscopy, 53, 960-964.

Cremers, D. A., \& Radziemski, L. J. (2013). Handbook of laserinduced breakdown spectroscopy. Oxford, England: John Wiley \& Sons Ltd.

Cristoforetti, G., De Giacomo, A., Dell'Aglio, M., Legnaioli, S., Tognoni, E., Palleschi, V., \& Omenetto, N. (2010). Local thermodynamic equilibrium in laser-induced breakdown spectroscopy:
Beyond the McWhirter criterion. Spectrochimica Acta Part B, 65, $86-95$.

Curcio, D., Ciraolo, G., D'Asaro, F., \& Minacapilli, M. (2013). Prediction of soil texture distributions using VNIR-SWIR reflectance spectroscopy. Procedia Environmental Sciences, 19, 494-503.

Dawson, J. B., Snook, R. D., \& Price, W. J. (1993). Background and background correction in analytical atomic spectrometry. Part 1. Emission spectrometry. A tutorial review. Journal of Analytical Atomic Spectrometry, 8, 517-537.

Day, D., Connors, B., Jennings, M., Egan, J., Derman, K., Soucy, P., ... Sackett, D. (2015). A full featured handheld LIBS analyzer with early results for defense and security. Proc. SPIE, Next-Generation Spectroscopic Technologies VIII, 9482, 948206. Baltimore, Maryland: SPIE Sensing Technology + Applications. https://doi.org/10. $1117 / 12.2177565$

Day, D. R., Derman, K., Egan, J. F., \& Soucy, P. E. (2015). Handheld LIBS analyzer end plate purging structure. US Patent 9,395,243 B2.

Deming, S. N., Palasota, J. A., \& Palasota, J. M. (1991). Experimental design in chemometrics. Journal of Chemometrics, 5, 181-192.

Díaz, D., Hahn, D. W., \& Molina, A. (2012). Evaluation of laserinduced breakdown spectroscopy (LIBS) as a measurement technique for evaluation of total elemental concentration in soils. Applied Spectroscopy, 66, 99-106.

Eppler, A. S., Cremers, D. A., Hickmott, D. D., Ferris, M. J., \& Koskelo, A. C. (1996). Matrix effects in the detection of $\mathrm{Pb}$ and $\mathrm{Ba}$ in soils using laser-induced breakdown spectroscopy. Applied Spectroscopy, 50, 1175-1181.

Falkowski, P. (2000). The global carbon cycle: A test of our knowledge of earth as a system. Science, 290, 291-296.

Ferreira, E. C., Anzano, S. M., Milori, D. M. B. P., Ferreira, E. J., Lasheras, R. J., Bonilla, B., ... Martin Neto, L. (2009). Multiple response optimization of laser-induced breakdown spectroscopy parameters for multi-element analysis of soil samples. Applied Spectroscopy, 63, 1081-1088.

Ferreira, E. C., Ferreira, E. J., Villas-Boas, P. R., Senesi, G. S., Carvalho, C. M., Romano, R. A., ... Milori, D. M. B. P. (2014). Novel estimation of the humification degree of soil organic matter by laser-induced breakdown spectroscopy (LIBS). Spectrochimica Acta Part B, 99, 76-81.

Ferreira, E. C., Gomes Neto, J. A., Milori, D. M. B. P., Ferreira, E. J., \& Anzano, J. M. (2015). Laser-induced breakdown spectroscopy: Extending its application to soil $\mathrm{pH}$ measurements. Spectrochimica Acta Part B, 110, 96-99.

Ferreira, E. C., Milori, D. M. B. P., Ferreira, E. J., Da Silva, R. M., \& Martin-Neto, L. (2008). Artificial neural network for $\mathrm{Cu}$ quantitative determination in soil using a portable laser induced breakdown spectroscopy system. Spectrochimica Acta Part B, 63, 1216-1220.

Ferreira, E. C., Milori, D. M. B. P., Ferreira, E. J., Dos Santos, L. M., Martin-Neto, L., Nogueira, A. R. D. A., ... Nogueira, A. R. D. A. (2011). Evaluation of laser induced breakdown spectroscopy for multielemental determination in soils under sewage sludge application. Talanta, 85, 435-440.

Gornushkin, I. B., Shabanov, S. V., Merk, S., Tognoni, E., \& Panne, U. (2010). Effects of non-uniformity of laser induced plasma on plasma temperature and concentrations determined by the Boltzmann plot method: Implications from plasma modeling. Journal of Analytical Atomic Spectrometry, 25, 1643-1653. 
Grotzinger, J. P., Crisp, J., Vasavada, A. R., Anderson, R. C., Baker, C. J., Barry, R., ... Wiens, R. C. (2012). Mars science laboratory mission and science investigation. Space Science Reviews, $170,5-56$

Hahn, D. W., \& Omenetto, N. (2012). Laser-induced breakdown spectroscopy (LIBS), part II: Review of instrumental and methodological approaches to material analysis and applications to different fields. Applied Spectroscopy, 66, 347-419.

Hahn, W. D., \& Omenetto, N. (2010). Laser-induced breakdown spectroscopy (LIBS), part I: Review of basic diagnostics and plasma? Particle interactions: Still-challenging issues within the analytical plasma community. Applied Spectroscopy, 64, 335A-366A.

Harmon, R. S., Russo, R. E., \& Hark, R. R. (2013). Applications of laser-induced breakdown spectroscopy for geochemical and environmental analysis: A comprehensive review. Spectrochimica Acta Part B, 87, 11-26.

Harris, R. D., Cremers, D. A., Ebinger, M. H., \& Bluhm, B. K. (2004). Determination of nitrogen in sand using laser-induced breakdown spectroscopy. Applied Spectroscopy, 58, 770-775.

Hilbk-Kortenbruck, F., Noll, R., Wintjens, P., Falk, H., \& Becker, C. (2001). Analysis of heavy metals in soils using laser-induced breakdown spectrometry combined with laser-induced fluorescence. Spectrochimica Acta Part B, 56, 933-945.

Houba, V. J. G., Temminghoff, E. J. M., Gaikhorst, G. A., \& van Vark, W. (2000). Soil analysis procedures using $0.01 M$ calcium chloride as extraction reagent. Communications in Soil Science and Plant Analysis, 31, 1299-1396.

Huang, C. L., \& Schulte, E. E. (1985). Digestion of plant tissue for analysis by ICP emission spectroscopy. Communications in Soil Science and Plant Analysis, 16, 943-958.

Jantzi, S. C., Motto-Ros, V., Trichard, F., Markushin, Y., Melikechi, N., \& De Giacomo, A. (2016). Sample treatment and preparation for laser-induced breakdown spectroscopy. Spectrochimica Acta Part B, 115, 52-63.

Knadel, M., Gislum, R., Hermansen, C., Peng, Y., Moldrup, P., de Jonge, L. W., \& Greve, M. H. (2017). Comparing predictive ability of laser-induced breakdown spectroscopy to visible near-infrared spectroscopy for soil property determination. Biosystems Engineering, 156, 157-172.

Kramida, A., Ralchenko, Y., Reader, J., \& NIST ASD Team. (2018). NIST atomic spectra database (version 5.5.3) (Online). Retrieved from https://physics.nist.gov/asd

Lee, W., Wu, J., Lee, Y., \& Sneddon, J. (2004). Recent applications of laser-induced breakdown spectrometry: A review of material approaches. Applied Spectroscopy Reviews, 39, 27-97.

Liland, K. H. (2015). 4S peak filling - baseline estimation by iterative mean suppression. MethodsX, 2, 135-140.

Liland, K. H., Almøy, T., \& Mevik, B.-H. (2010). Optimal choice of baseline correction for multivariate calibration of spectra. Applied Spectroscopy, 64, 1007-1016.

Liland, K. H., \& Mevik, T. A. B.-H. (2011). Optimal baseline correction for multivariate calibration using open-source software. Life Science Instruments, 3, 7.

Liu, T. K., Odell, R. T., Etter, W. C., \& Thornburn, T. H. (1966). A comparison of clay contents determined by hydrometer and pipette methods using reduced major Axis Analysis ${ }^{1}$. Soil Science Society of America Journal, 30, 665.

Madari, B. E., Reeves, J. B., Machado, P. L. O. A., Guimarães, C. M., Torres, E., \& McCarty, G. W. (2006). Mid- and near-infrared spectroscopic assessment of soil compositional parameters and structural indices in two Ferralsols. Geoderma, 136, 245-259.

McCave, I. N., \& Syvitski, J. P. M. (1991). Principles and methods of geological particle size analysis. In J. P. M. Syvitski (Ed.), Principles, methods and application of particle size analysis (pp. 3-21). Cambridge, England: Cambridge University Press.

McLean, E. O. (1982). Soil pH and lime requirement. In A. L. Page, R. H. Miller, \& D. R. Keeney (Eds.), Methods of soil analysis. Part 2. Chemical and microbiological properties (2nd ed., pp. 199-224). Madison, WI: American Society of Agronomy, Soil Science Society of America.

Mebane, W. R., Jr., \& Sekhon, J. S. (2011). Genetic optimization using derivatives: The rgenoud package for R. Journal of Statistical Software, 42, 1-26.

Mehlich, A. (1984). Mehlich 3 soil test extractant: A modification of Mehlich 2 extractant. Communications in Soil Science and Plant Analysis, 15, 1409-1416.

Milori, D. M. B. P., Galeti, H. V. A., Martin-Neto, L., Dieckow, J., González-Pérez, M., Bayer, C., \& Salton, J. (2006). Organic matter study of whole soil samples using laser-induced fluorescence spectroscopy. Soil Science Society of America Journal, $70,57$.

Miziolek, A. W., Palleschi, V., \& Schechter, I. (2006). Laser-induced breakdown spectroscopy (LIBS): Fundamentals and applications. New York, NY: Cambridge University Press.

Naime, J. M., Vaz, C. M. P., \& Macedo, A. (2001). Automated soil particle size analyzer based on gamma-ray attenuation. Computers and Electronics in Agriculture, 31, 295-304.

Nicolodelli, G., Marangoni, B. S., Cabral, J. S., Villas-Boas, P. R., Senesi, G. S., Dos Santos, C. H., ... Milori, D. M. B. P. (2014). Quantification of total carbon in soil using laser-induced breakdown spectroscopy: a method to correct interference lines. Applied Optics, 53, 2170-2176.

Noll, R. (2012). Laser-induced breakdown spectroscopy: Fundamentals and applications. New York, NY: Springer.

Olsen, S. R., Cole, C. V., Watanabe, F. S., \& Dean, L. A. (1954). Estimation of available phosphorus in soils by extraction with sodium bicarbonate. Washington, DC: U.S. Government Printing Office.

Pontes, M. J. C., Cortez, J., Galvão, R. K. H., Pasquini, C., Araújo, M. C. U., Coelho, R. M., ... Madari, B. E. (2009). Classification of Brazilian soils by using LIBS and variable selection in the wavelet domain. Analytica Chimica Acta, 642, 12-18.

Sallé, B., Cremers, D. A., Maurice, S., Wiens, R. C., \& Fichet, P. (2005). Evaluation of a compact spectrograph for in-situ and standoff laser-induced breakdown spectroscopy analyses of geological samples on Mars missions. Spectrochimica Acta Part B, 60, 805-815.

Santos, D., Nunes, L. C., Trevizan, L. C., Godoi, Q., Leme, F. O., Braga, J. W. B., \& Krug, F. J. (2009). Evaluation of laser induced breakdown spectroscopy for cadmium determination in soils. Spectrochimica Acta Part B, 64, 1073-1078.

Saxton, K. E., Rawls, W. J., Romberger, J. S., \& Papendick, R. I. (1986). Estimating generalized soil-water characteristics from texture. Soil Science Society of America Journal, 50, 1031-1036.

Schofield, R. K., \& Taylor, A. W. (1955). The measurement of soil pH. Soil Science Society of America Journal, 19, 164-167. 
Segnini, A., Xavier, A. A. P., Otaviani-junior, P. L., Ferreira, E. C., Watanabe, A. M., Sperança, M. A., ... Milori, D. M. B. P. (2014). Physical and chemical matrix effects in soil carbon quantification using laser-induced breakdown spectroscopy. American Journal of Analytical Chemistry, 05, 722-729.

Senesi, G. S., Dell'Aglio, M., Gaudiuso, R., De Giacomo, A., Zaccone, C., De Pascale, O., ... Capitelli, M. (2009). Heavy metal concentrations in soils as determined by laser-induced breakdown spectroscopy (LIBS), with special emphasis on chromium. Environmental Research, 109, 413-420.

Senesi, G. S., Martin-Neto, L., Villas-Boas, P. R., Nicolodelli, G., \& Milori, D. M. B. P. (2018). Laser-based spectroscopic methods to evaluate the humification degree of soil organic matter in whole soils: A review. Journal of Soils and Sediments, 18, 1292-1302.

Senesi, G. S., \& Senesi, N. (2016). Laser-induced breakdown spectroscopy (LIBS) to measure quantitatively soil carbon with emphasis on soil organic carbon. A review. Analytica Chimica Acta, 938, 7-17.

Smith, P. L., Heise, C., Esmond, J. R., \& Kurucz, R. L. (1995). Atomic spectral line database, built from atomic data files from R.L. Kurucz' CD-ROM 23. Retrieved from http://www.pmp.unihannover.de/cgi-bin/ssi/test/kurucz/sekur.html

Tejada, M., \& Gonzalez, J. L. (2006). Crushed cotton gin compost on soil biological properties and rice yield. European Journal of Agronomy, 25, 22-29.

Thompson, M., \& Barnes, R. M. (1992). Analytical performance of inductively coupled plasma -Atomic emission spectrometry. In A. Monstaser \& D. W. Golightly (Eds.), Inductively coupled plasmas in analytical atomic spectrometry (2nd ed., pp. 249-247). New York, NY: Wiley-VHC.

Tüzen, M. (2003). Determination of heavy metals in soil, mushroom and plant samples by atomic absorption spectrometry. Microchemical Journal, 74, 289-297.

Villas-Boas, P. R., Franco, M. A., Gollany, H. T., Martin-Neto, L., \& Milori, D. M. B. P. (2019). Applications of laser-induced breakdown spectroscopy for soil characterization, Part II: Review of elemental analysis and soil classification. European Journal of Soil Science.
Villas-Boas, P. R., Romano, R. A., de Menezes Franco, M. A., Ferreira, E. C., Ferreira, E. J., Crestana, S., \& Milori, D. M. B. P. (2016). Laser-induced breakdown spectroscopy to determine soil texture: A fast analytical technique. Geoderma, 263, 195-202.

Viscarra Rossel, R. A., Walvoort, D. J. J., McBratney, A. B., Janik, L. J., \& Skjemstad, J. O. (2006). Visible, near infrared, mid infrared or combined diffuse reflectance spectroscopy for simultaneous assessment of various soil properties. Geoderma, 131, 59-75.

Yamamoto, K. Y., Cremers, D. A., Ferris, M. J., \& Foster, L. E. (1996). Detection of metals in the environment using a portable laser-induced breakdown spectroscopy instrument. Applied Spectroscopy, 50, 222-233.

Yeomans, J. C., \& Bremner, J. M. (1991). Carbon and nitrogen analysis of soils by automated combustion techniques. Communications in Soil Science and Plant Analysis, 22, 843-850.

Zhang, T. L., Wu, S., Tang, H. S., Wang, K., Duan, Y. X., \& Li, H. (2015). Progress of chemometrics in laser-induced breakdown spectroscopy analysis. Chinese Journal of Analytical Chemistry, 43, 939-948.

Zorov, N. B., Gorbatenko, A. A., Labutin, T. A., \& Popov, A. M. (2010). A review of normalization techniques in analytical atomic spectrometry with laser sampling: From single to multivariate correction. Spectrochimica Acta Part B, 65, 642-657.

Zorov, N. B., Popov, A. M., Zaytsev, S. M., \& Labutin, T. A. (2015). Qualitative and quantitative analysis of environmental samples by laser-induced breakdown spectrometry. Russian Chemical Reviews, $84,1021-1050$.

How to cite this article: Villas-Boas PR, Franco MA, Martin-Neto L, Gollany HT, Milori DMBP. Applications of laser-induced breakdown spectroscopy for soil analysis, part I: Review of fundamentals and chemical and physical properties. Eur J Soil Sci. 2019;1-16. https://doi.org/ $\underline{10.1111 / \text { ejss. } 12888}$ 